\title{
Increasing Adult Hippocampal Neurogenesis is Sufficient to Reduce Anxiety and Depression-Like Behaviors
}

\author{
Alexis S Hill', Amar Sahay ${ }^{*, 2,3,4}$ and René Hen ${ }^{*, 1,5,6,7}$ \\ 'Department of Neuroscience, Columbia University, New York, NY, USA; '2Center for Regenerative Medicine, Department of Psychiatry, \\ Massachusetts General Hospital, Boston, MA, USA; ${ }^{3}$ Harvard Stem Cell Institute, Boston, MA, USA; ${ }^{4}$ Harvard Medical School, Boston, MA, USA; \\ ${ }^{5}$ Department of Psychiatry, Columbia University, New York, NY, USA; ${ }^{6}$ Department of Pharmacology, Columbia University, New York, NY, USA; \\ ${ }^{7}$ Division of Integrative Neuroscience, The New York State Psychiatric Institute, New York, NY, USA
}

\begin{abstract}
Adult hippocampal neurogenesis is increased by antidepressants, and is required for some of their behavioral effects. However, it remains unclear whether expanding the population of adult-born neurons is sufficient to affect anxiety and depression-related behavior. Here, we use an inducible transgenic mouse model in which the pro-apoptotic gene Bax is deleted from neural stem cells and their progeny in the adult brain, and thereby increases adult neurogenesis. We find no effects on baseline anxiety and depression-related behavior, however, we find that increasing adult neurogenesis is sufficient to reduce anxiety and depression-related behaviors in mice treated chronically with corticosterone (CORT), a mouse model of stress. Thus, neurogenesis differentially affects behavior under baseline conditions and in a model of chronic stress. Moreover, we find no effect of increased adult hippocampal neurogenesis on hypothalamic-pituitary-adrenal (HPA) axis regulation, either at baseline or following chronic CORT administration, suggesting that increasing adult hippocampal neurogenesis can affect anxiety and depression-related behavior through a mechanism independent of the HPA axis. The use of future techniques to specifically inhibit BAX in the hippocampus could be used to augment adult neurogenesis, and may therefore represent a novel strategy to promote antidepressant-like behavioral effects.

Neuropsychopharmacology (20I5) 40, 2368-2378; doi:I0.I038/npp.20I5.85; published online I5 April 2015
\end{abstract}

\section{INTRODUCTION}

Adult hippocampal neurogenesis is a process through which additional granule cells are added to the dentate gyrus throughout life. These cells are produced from progenitors located in the subgranular zone of the dentate gyrus, and their rates of proliferation, maturation, and survival are impacted by environmental conditions such as age, stress, exercise, and antidepressants (Dranovsky et al, 2011; Gould et al, 1997; Malberg et al, 2000; van Praag et al, 2005).

Many antidepressants are known to act through monoamine systems, however the downstream mechanisms through which they affect mood are still not entirely understood. While many antidepressants change monoamine levels within hours, effective changes in mood are not seen for 3-4 weeks. This disparity, along with the findings that antidepressants increase the number of adult-born neurons (Boldrini et al, 2009; Malberg et al, 2000), which take about 4 weeks to form

*Correspondence: Dr A Sahay, Center for Regenerative Medicine, Department of Psychiatry, Massachusetts General Hospital, 185 Cambridge Street, CPZN-Room 4242, Boston, MA 02I I4, USA, Tel: + I 617643 437।, Fax: + 617724 2662, E-mail: asahay@mgh.harvard.edu or Professor R Hen, Center for Neurobiology and Behavior, Columbia University, I05I Riverside Drive, Unit 87, Kolb Annex, Room 767, New York, NY I0032, USA, Tel: + I 646774 7I07, Fax: + I 2125435074 , E-mail: rh95@columbia.edu

Received 21 December 2014; revised 13 March 2015; accepted 17 March 2015; accepted article preview online 2 April 2015 synaptic connections (Toni et al, 2007) and contribute to behavior in rodents (Denny et al, 2012; Kee et al, 2007), led to the hypothesis that antidepressants might affect mood by increasing adult hippocampal neurogenesis (Duman et al, 2001). Since these initial observations, adult hippocampal neurogenesis has been shown to be required for some, but not all, of the behavioral effects of antidepressants (David et al, 2009; Santarelli et al, 2003). However, one important remaining question is whether increasing levels of adult hippocampal neurogenesis is sufficient for antidepressantlike effects on behavior. Tangential support for this hypothesis includes a study in which deletion of neurofibromin 1 from adult-born cells increased levels of adult neurogenesis and had an antidepressant-like effect on behavior; however, this manipulation not only increased the number of adultborn neurons, but also affected these cells in other ways, such as by activating ERK signaling ( $\mathrm{Li}$ et al, 2012). More recently, the P7C3 compound has been shown to increase adult neurogenesis and has an antidepressant-like effect on social interaction behavior following social defeat; however, P7C3 likely exerts its effects through multiple biological processes including, but not limited to, neurogenesis (Walker et al, 2014; Wang et al, 2014; Yin et al, 2014).

To directly assess the effects of selectively increasing the number of adult-born neurons, we previously generated a transgenic mouse line with increased adult hippocampal neurogenesis (Sahay et al, 2011). Under baseline conditions, increased adult neurogenesis enhances pattern separation, 
but does not impact anxiety or depression-related behavior (Sahay et al, 2011). Here, we show that increasing adult hippocampal neurogenesis using this same transgenic mouse model is sufficient to promote resilience to chronic corticosterone (CORT) administration, a model of stress. These findings extend our understanding of the contribution of adult neurogenesis to anxiety and depression, and suggest that neurogenesis may be harnessed to develop novel antidepressants.

\section{MATERIALS AND METHODS}

\section{Mice}

iBax mice are homozygous for a loxP-flanked Bax allele (Takeuchi et al, 2005), hemizygous for a Nestin-CreERT2 transgene (Dranovsky et $a l, 2011$ ), and maintained on a mixed C57BL/6 and 129/SvEv background, as previously described (Sahay et al, 2011). Although some ectopic expression has been reported in this transgenic line (Sun et al, 2014), it is unlikely to influence our phenotype since these ectopic cells are not undergoing apoptosis, and are therefore unlikely to be affected by the Bax deletion. Mice were housed two to five per cage and maintained on a $12 \mathrm{~h}$ light/dark schedule with continuous access to food and water. All behavioral testing was conducted during the light cycle with approval from the Institutional Animal Care and Use Committees at both Columbia University and the New York State Psychiatric Institute. All mice used in experiments reported here are males.

\section{Drug Administration}

Tamoxifen (TAM) was dissolved in a solution of corn oil (C8267, Sigma, St Louis, MO) and 10\% ethanol. iBax mice (8-10-week-old) received $2 \mathrm{mg}$ TAM (10 mg/ml, Sigma), or the same volume of corn oil and ethanol (vehicle), intraperitoneally once per day for 5 consecutive days. This dose of TAM has previously been shown to have no effect on behavior in iBax mice (Sahay et al, 2011).

CORT (C2505, Sigma) was dissolved in .45\% betacyclodextrin, administered ad libitum in opaque bottles, and replaced every 3-4 days. iBax mice received $70 \mu \mathrm{g} / \mathrm{ml}$ CORT, equivalent to $10 \mathrm{mg} / \mathrm{kg} /$ day, an effective dose in this strain (unpublished data).

Bromodeoxyuridine (BrdU) (150 mg/kg body weight, dissolved in $0.9 \% \mathrm{NaCl}$ (Roche, Indianapolis, IN)) was injected intraperitoneally once per day for 2 days at designated time points.

\section{Behavioral Testing}

Mice were tested in the open field (MED Associates, St Albans, VT), a square $43.2 \times 43.2 \mathrm{~cm}$ enclosure with infrared beams to detect animal movement, for $30 \mathrm{~min}$. The center region was defined by the inner $21.2 \times 21.2 \mathrm{~cm}$ area. Total distance, percent center distance (the percent of the total distance that occurs in the center region), time in center, and center entries were analyzed by MED Associates software.

Mice were tested in the elevated plus maze for $5 \mathrm{~min}$. The maze used has arms $7.6-\mathrm{cm}$ wide, $28-\mathrm{cm}$ long, and $31 \mathrm{~cm}$ above the floor. Two opposing closed arms have $15 \mathrm{~cm}$ walls, while two opposing open arms have a $1 \mathrm{~cm}$ lip. Testing was video recorded and later analyzed by an observer (blind to genotype) for time spent and entries into the open arms.

In the tail suspension test, mice were suspended from a table-top by their tails for $5 \mathrm{~min}$. This test was video recorded and mobility was analyzed using ViewPoint Life Sciences software (ViewPoint, Montréal, Canada).

The forced swim test was conducted as in the study by Sahay et al (2011) for $6 \mathrm{~min}$ on each of 2 days, with the first day serving the purpose of pre-exposure. Mobility on the second day was analyzed using ViewPoint Life Sciences Software.

In preparation for the novelty-suppressed feeding test, mice were food deprived in their home cages for $24-26 \mathrm{~h}$ before testing. The testing apparatus consisted of a plastic arena (45- $\mathrm{cm}$ long, $15-\mathrm{cm}$ high, and $30 \mathrm{~cm}$ wide) with the floor covered by $2 \mathrm{~cm}$ of wood-chip bedding. A single food pellet (familiar laboratory mouse chow) was placed on circular white filter paper $(12 \mathrm{~cm}$ in diameter) in the center of the arena. Mice were placed in a corner of the arena, and the latency to begin feeding on the food pellet was recorded (up to a maximum time of $10 \mathrm{~min}$ ). Testing was conducted under bright light conditions.

\section{Plasma CORT Levels}

In the no-stress experiment (Figure 2), hypothalamicpituitary-adrenal (HPA) axis activity was assessed in two paradigms. In the first, mice were placed in a decapicone plastic restraining device (Braintree Scientific, Braintree, MA) for $30 \mathrm{~min}$, and then placed in a novel cage (as in the study by Snyder et al (2011)). Blood was taken from different groups of mice for each time point: from the home cage (before restraint), immediately after restraint, or after 30 or $60 \mathrm{~min}$ in a novel cage following restraint. Blood was collected via submandibular bleed using a Goldenrod Animal Lancet (MEDIpoint, Mineola, NY), into a tube containing EDTA. Blood was centrifuged at $2000 \mathrm{rpm}$ for $5 \mathrm{~min}$ for separation of plasma, which was stored at $-20^{\circ} \mathrm{C}$ until assayed using an EIA kit (Arbor Assays, Ann Arbor, MI).

In the second paradigm, mice were placed in a novel cage without bedding for $15 \mathrm{~min}$ (as in the study by Schloesser et al (2009)). Blood was collected immediately afterwards, and treated as described above.

To examine HPA axis response to acute stress in the chronic CORT model (Figure 4e), mice were subjected to a $1 \mathrm{~min}$ swim stress, where animals were placed in a beaker of room temperature water. Trunk blood was collected 5 min later (as in the study by David et al (2009)) and treated as above.

\section{Immunohistochemistry}

At sacrifice, mice were anesthetized with ketamine and xylazine $(100 \mathrm{mg} / \mathrm{kg}$ and $7 \mathrm{mg} / \mathrm{kg}$, respectively) and transcardially perfused with cold saline and $4 \%$ paraformaldehyde. Brains were postfixed in $4 \%$ paraformaldehyde overnight, transferred to $30 \%$ sucrose for cryoprotection, and coronally sectioned $(35 \mu \mathrm{m})$.

For BrdU, immunostaining was conducted on slides as in the study by Sahay et al (2011) with rat anti-BrdU (OBT0030 Serotec, Raleigh, NC) and donkey anti-rat Cy3 (Jackson ImmunoResearch, Westgrove, PA) antibodies. Analysis was conducted using an Axiovert 200 microscope (Zeiss, 
Oberkochen, Germany) by an investigator blind to treatment.

Doublecortin (DCX) immunostaining was conducted as in the study by Sahay et al (2011) with goat anti-DCX (SC-8066, Santa Cruz Biotechnology, Santa Cruz, CA) and biotin donkey anti-goat (Jackson ImmunoResearch) antibodies, followed by avidin/biotin (ABC kit, Vector Laboratories, Burlingame, CA), and DAB peroxidase (Vector Laboratories). An investigator blind to treatment counted every sixth section throughout the dentate gyrus for the total number of DCX-positive neurons and for those with tertiary dendrites. Analysis was conducted using an Axio Observer A.1 microscope (Zeiss).

\section{Statistical Methods}

Due to animal number constraints, we were unable to test all four groups (control, TAM, CORT, and TAM+CORT) concurrently, and therefore instead tested vehicle and TAM in one experiment, and vehicle, CORT, and TAM+CORT in a second experiment. One-way ANOVAs were used to assess significance of comparisons between vehicle and TAM in the first experiment, and between vehicle and CORT as well as between CORT and TAM+CORT in the second experiment, which were the comparisons we were interested in a priori. To statistically assess main effects of TAM and CORT, or interactions between the two variables, we combined and normalized data from these two experiments, as depicted in Supplementary Figure 1. To do this, for each behavioral measure, controls from each test were normalized to a $0-10$ scale using unity base normalization, and then the other groups in each experiment were normalized accordingly. The following equation was used: $[a+(x-A)(b-a)] /(B-A)$ where $A=$ minimum value in the control group, $B=$ maximum value in the control group, $a=0$ (normalized minimum), $b=10$ (normalized maximum), and $x=$ the data point to be normalized.

To analyze the effects of two variables in the novelty suppressed feeding test, data were analyzed using the Cox Proportional Hazard model, using SAS software (SAS Institute, Cary, NC). The effects of single variables in this test were analyzed using Kaplan-Meier Survival Analysis. All other statistical analyses were conducted using StatView software (SAS Institute). Results were considered statistically significant if $P<0.05$.

Extended statistical details for all experiments are included in Supplementary Table 1.

\section{RESULTS}

\section{Genetic Deletion of Bax Increases Adult Hippocampal Neurogenesis, but does not Affect Anxiety and Antidepressant-Responsive Behavior Under No-Stress Conditions}

To genetically increase levels of adult neurogenesis, we utilized a bi-transgenic mouse model that expresses the TAM-dependent recombinase CreERT2 under control of the Nestin promoter, as well as floxed alleles of the pro-apoptotic gene Bax. In this mouse line, TAM treatment allows inducible deletion of Bax in neural stem cells and their progeny (iBax mice). Normally, $60-80 \%$ of adult-born dentate granule neurons undergo cell death before reaching maturity through a process that requires BAX (Dayer et al, 2003). This transgenic design increases neurogenesis by preventing cell death in the adult-born neuronal population (Sahay et al, 2011) (Figure 1a).

Changes in neurogenesis were assessed after sacrifice by the number of cells labeled with the thymidine-analog BrdU, which was injected the week following TAM induction, and becomes incorporated into cells that are dividing at the time of injection (Figure 1b). As previously reported (Sahay et al, 2011), we observed a large increase in the number of BrdUlabeled cells in the dentate gyrus in mice treated with TAM as compared with vehicle. Here, at 8 weeks post-BrdU injection, we observed a fourfold increase in the total number of BrdU-positive cells $(\mathrm{F}(1,8)=12.844, P<0.01)$ (Figure 1c), an increase seen in both dorsal (3.7-fold increase, $F(1,8)=$ 16.595, $P<0.01)$, and ventral $(4.5$-fold increase, $F(1,8)=$ $8.968, P<0.05)$ dentate gyrus.

While neurons make up the majority of adult-born cells labeled with BrdU in the hippocampus, stem cells, and astrocytes and potentially even oligodendrocytes (Chetty et $a l, 2014)$ are also found. To determine the proportion of neurons produced, colabeling was assessed between BrdU and the mature neuronal marker neuronal nuclei (NeuN). We observed no significant differences between groups in the proportion of BrdU labeled cells that were colabeled with NeuN, when assessed throughout the entire dentate gyrus, or in either the dorsal or ventral subregions $(F(1,4)<1.056$, $P>0.05)$ (Figure 1c).

Recently, studies have suggested the presence of adultneurogenesis in the hypothalamus (Batailler et al, 2014; Saaltink et al, 2012), involved in maintaining weight and metabolism (Lee and Blackshaw, 2012). To determine

Figure I In the absence of stress, genetically increasing adult hippocampal neurogenesis in iBax mice increases adult hippocampal neurogenesis, but does not affect anxiety- and antidepressant-responsive behavior. (a) Schematic: vehicle-treated animals have normal levels of adult hippocampal neurogenesis where stem cells (red) can undergo symmetric or asymmetric division to produce mature granule cells (purple), however, the majority of produced cells undergo BAX-dependent cell death during maturation. Tamoxifen treatment in iBax mice prevents BAX-mediated cell death in recombined cells, leading to an increased number of adult-born granule cells. (b) Experimental timeline: iBax mice were injected with either vehicle (top line) or tamoxifen (TAM; bottom line) 6 weeks before behavioral testing. (c) TAM increases the total number of BrdU-positive cells in the dentate gyrus ( $P=0.007)$. Significant differences are

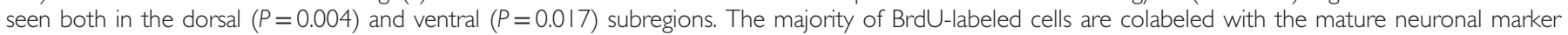

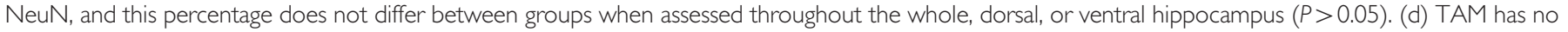

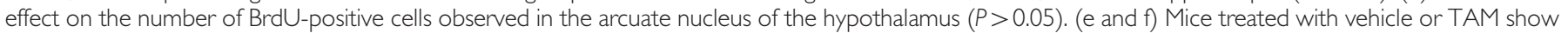

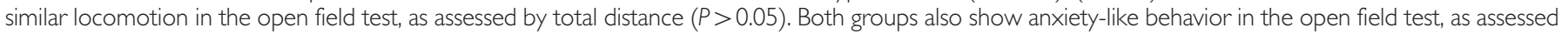
by percent center distance $(P>0.05)$, and in the elevated plus maze, as assessed by time spent in the open arms $(P>0.05)$ or open arm entries $(P>0.05)$.

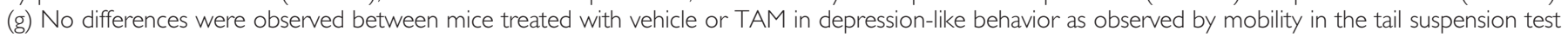

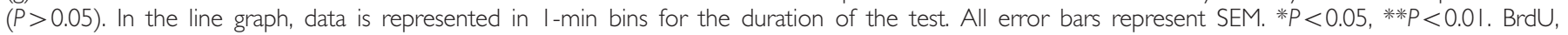
bromodeoxyuridine; NeuN, neuronal nuclei; TAM, Tamoxifen. 
whether hypothalamic adult neurogenesis is affected in $i B a x$ mice, we performed analysis of the number of BrdU-labeled cells in the arcuate nucleus, a portion of the hypothalamus where the presence of adult-born cells has been reported
(Kokoeva et al, 2005; Pierce and Xu, 2010; Xu et al, 2005). We observed no difference in the numbers of BrdU-labeled cells between vehicle- and TAM-treated mice $(\mathrm{F}(1,8)=1.694$, $P>0.05$ ) (Figure 1d). a

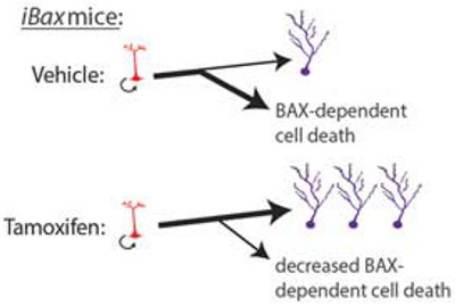

b

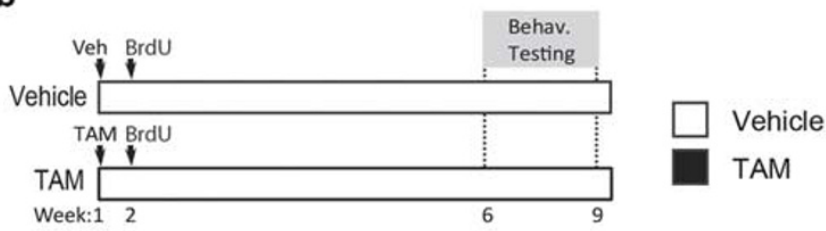

c
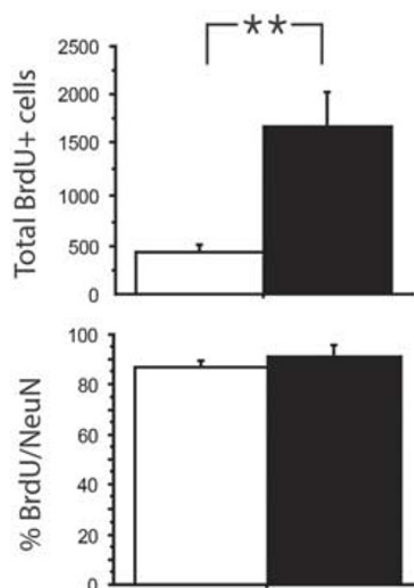

e
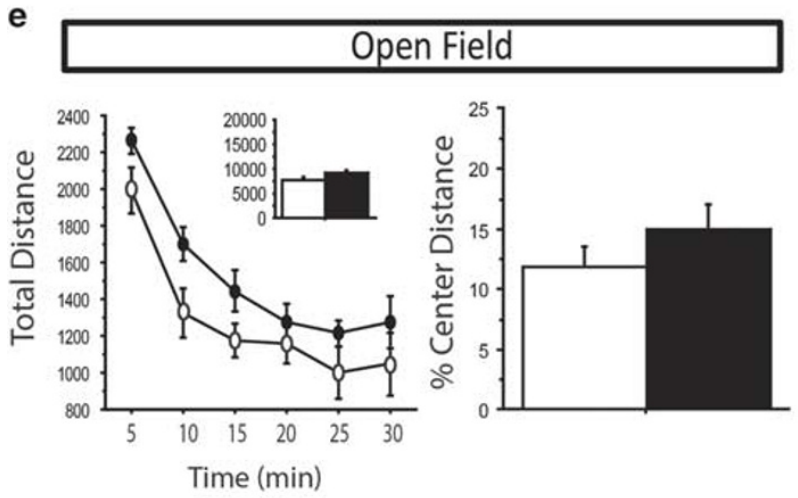

g
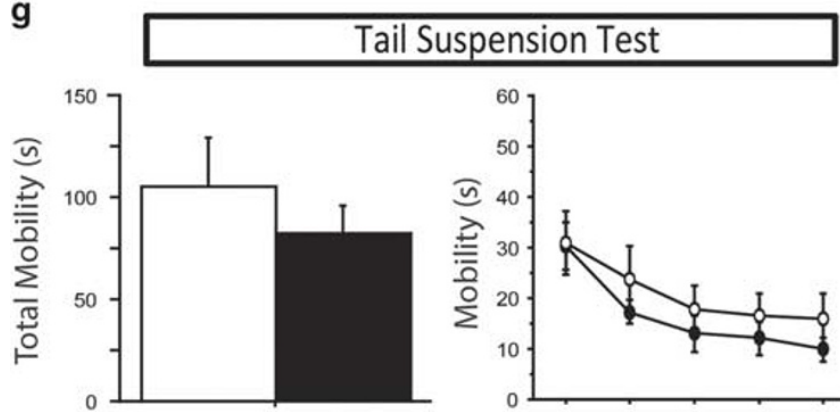

\section{Dentate Gyrus BrdU}
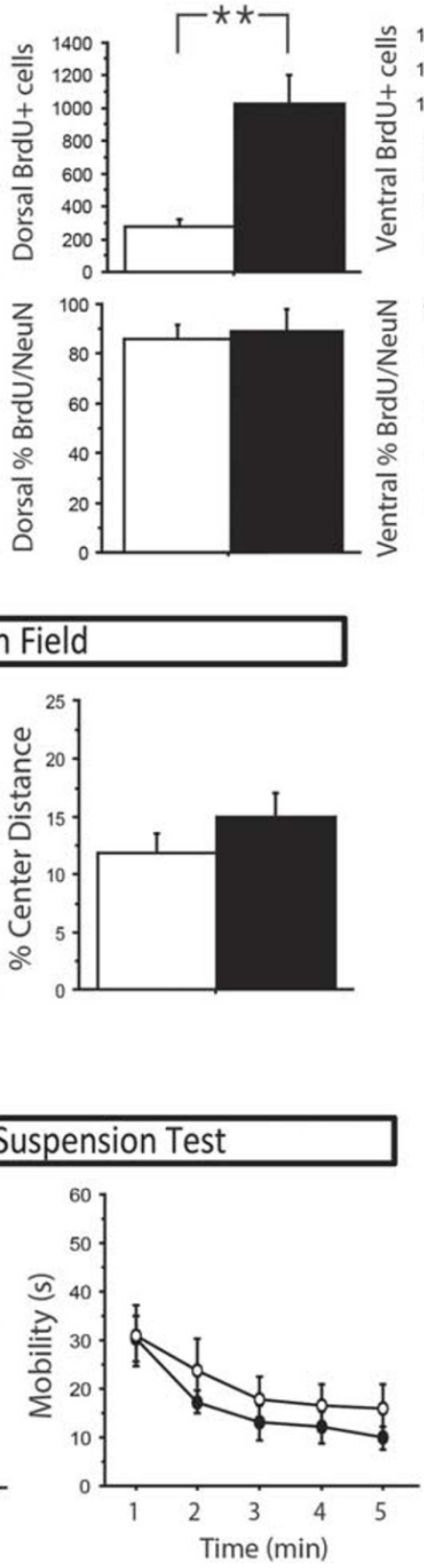
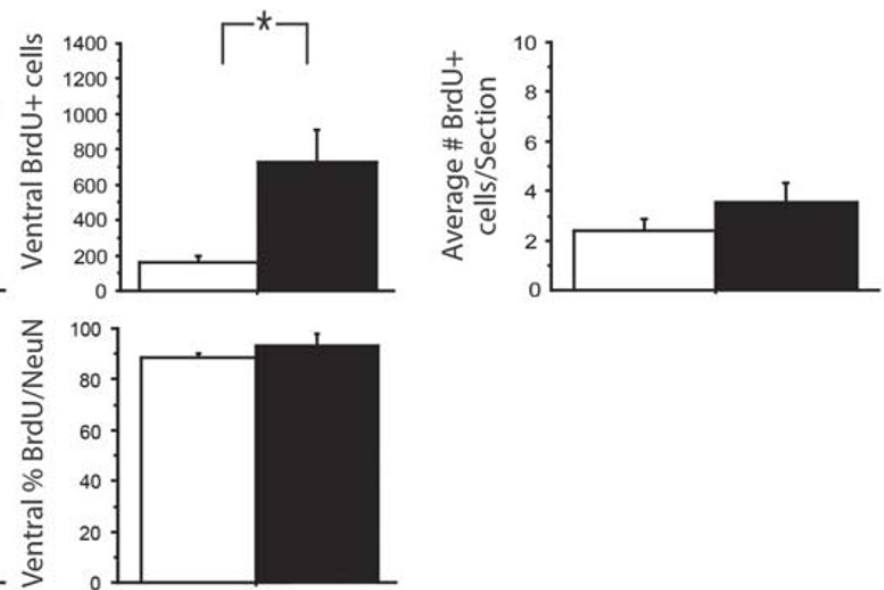

\section{f}

Elevated Plus Maze
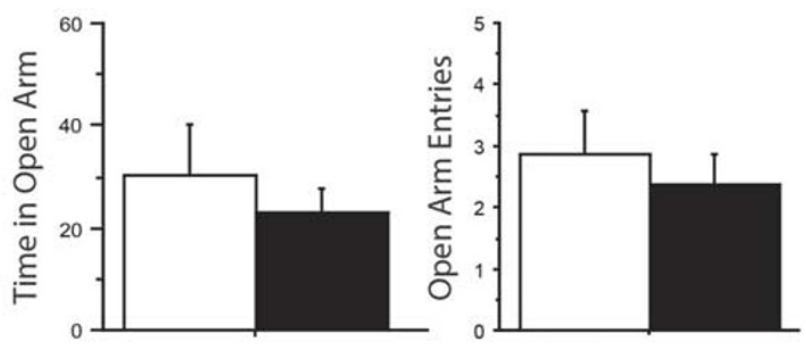
Initial characterization of this mouse model showed that under baseline, no-stress conditions, increasing adult neurogenesis enhances pattern separation, but has no effect on anxiety or depression-related behavior (Sahay et al, 2011). To confirm and extend this finding with additional behavioral tests, $i B a x$ mice were treated with either vehicle or TAM, and tested for anxiety and depression-related behavior 6 weeks later (Figure 1b). As expected, we observed no significant differences between vehicle- and TAM-treated groups in the open field, elevated plus maze, or tail suspension tests (Fs $<3.401, P>0.05$ ) (Figure $1 \mathrm{e}-\mathrm{g}$ ).

\section{Increasing Adult Hippocampal Neurogenesis does not Affect HPA Axis Response to Acute Stress}

Elimination of adult-hippocampal neurogenesis has been shown to impair regulation of the $\mathrm{HPA}$ axis, as several studies have now shown that mice with ablated adult neurogenesis have a heightened HPA axis response to acute stress (Schloesser et al, 2009; Snyder et al, 2011; Surget et al, 2011). We therefore initially hypothesized that increasing adult hippocampal neurogenesis might improve HPA axis regulation, which we expected to observe as decreased plasma CORT following acute stress. We tested whether this process is affected by increased adult neurogenesis, using the same paradigms which have been shown to require adult hippocampal neurogenesis for proper HPA axis regulation. Here, we observed no differences in plasma CORT between vehicle and TAM groups at baseline, following restraint stress, or following the mild stress of a novel cage (Fs $<2.154$, $P>0.05$ ) (Figure 2). Together, these results suggest that at baseline, increasing adult hippocampal neurogenesis is not sufficient to affect anxiety and depression-related behaviors or HPA axis regulation.

\section{Genetic Deletion of Bax in Adult Neural Stem Cells Prevents the Effects of Chronic CORT on Adult Neurogenesis}

It has been repeatedly shown that ablation of adult neurogenesis has no effect on anxiety- or depression-related behavior at baseline, but can prevent the enhancing effects of antidepressants and enriched environments (Santarelli et al, 2003; Schloesser et al, 2010). In addition, in some strains of mice, antidepressants have no effect at baseline, but do rescue the effects of chronic unpredictable mild stress (Surget et al, 2009) or chronic CORT (David et al, 2009). We therefore reasoned that even though increasing adult neurogenesis shows no effect on behavior at baseline, it might prevent the effects of stress. To test this hypothesis, we subjected iBax mice to chronic CORT (Figure 3a).

To determine the impact of genetic deletion of Bax on neurogenesis in mice treated with chronic CORT, we used immunohistochemistry. As in the previous experiment, mice were injected with BrdU the week following TAM induction. Here we observed no significant difference a

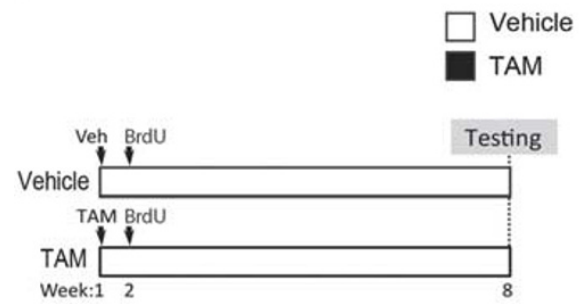

b
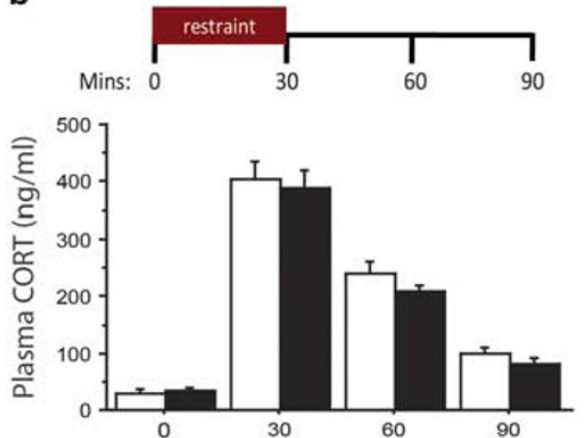
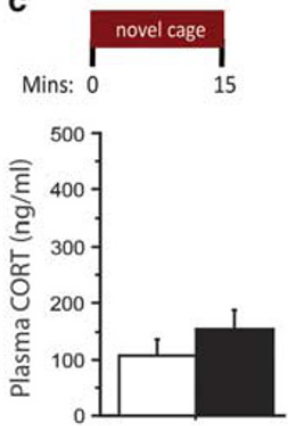

Figure 2 Genetically increasing adult hippocampal neurogenesis does not affect the HPA axis response to acute stress. (a) Experimental timeline. (b and c) There were no differences between vehicle and TAM groups in plasma CORT levels before or at various time points following 30 min of restraint stress or immediately following I 5 min in a novel cage $(P>0.05) . n=4-I$ I/group. All error bars represent SEM. CORT, corticosterone; HPA, hypothalamic-pituitaryadrenal; TAM, Tamoxifen.

Figure 3 Genetic ablation of Bax in neural stem cells and progenitors protects against chronic CORT-induced reduction in adult hippocampal neurogenesis (a) Experimental timeline: iBax mice were injected with either vehicle or TAM. Six weeks later, mice received vehicle or CORT via drinking water for the remainder of the experiment. Three behavioral groups were tested, vehicle (top line), CORT alone (middle line), and TAM+CORT (bottom line). (b and c) Representative images of BrdU and DCX in the dentate gyrus (scale bars 100 um). (d) While no significant difference in the number of BrdU-positive cells is observed between vehicle- and CORT-treated mice $(P>0.05)$, TAM+CORT treatment increases the number of BrdU-positive cells $(P=0.046)$ (dorsal $P=0.064$; ventral $P=0.027$ ). The majority of BrdU-labeled cells are colabeled with the mature neuronal marker NeuN. The percent of BrdU-labeled cells colabeled with NeuN is the same between all groups in the dorsal dentate gyrus. In the ventral dentate gyrus, a lower percentage of BrdU-positive cells are

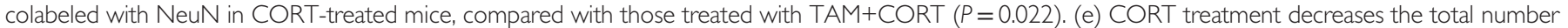
of DCX-positive neurons $(P=0.005)$, and there is a trend for a decrease in the number of DCX-positive neurons with tertiary dendrites $(P=0.07)$. TAM+CORT prevents these effects ( $P=0.002$ for total DCX-positive neurons; $P=0.002$ for DCX-positive neurons with tertiary dendrites). In the dorsal and ventral subregions, the total number of DCX-positive neurons is decreased by CORT (dorsal $P=0.07$; ventral $P=0.039$ ), and rescued in TAM+CORTtreated mice (dorsal $P=0.028$; ventral $P=0.002$ ); the number of DCX-positive neurons with tertiary dendrites is increased in TAM+CORT-treated mice compared with mice treated with CORT alone (dorsal $P=0.013$; ventral $P=0.00 \mathrm{I}$ ). $n=4-7$ /group in all analyses. All error bars represent SEM. * $P<0.05$, ** $P<0.01$. BrdU, bromodeoxyuridine; CORT, corticosterone; DCX, doublecortin; NeuN, neuronal nuclei; TAM, Tamoxifen. 
between vehicle- and CORT-treated mice $(\mathrm{F}(1,6)=$ 1.086, $P>0.05$ ) (Figure $3 \mathrm{~d}$ ). This was expected, as cells labeled at the time of BrdU injection were 4-weeks old at the onset of CORT treatment (see timeline Figure 3a), and therefore their number was unaffected by CORT. However, TAM+CORT animals had significantly greater

a

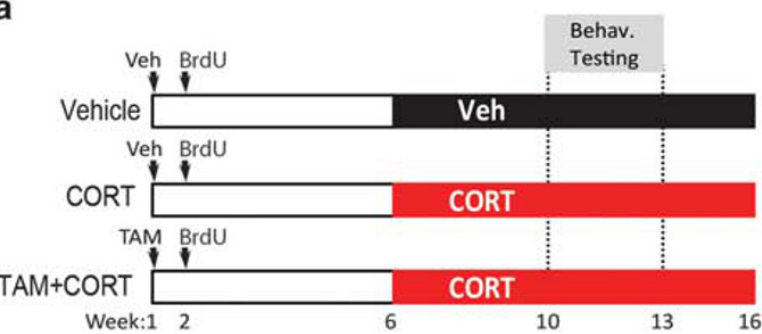

b

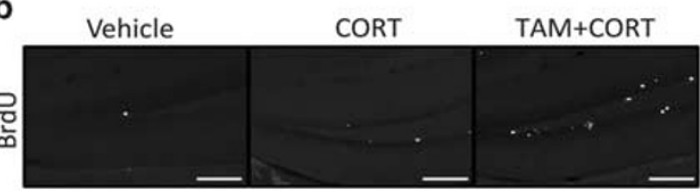

C

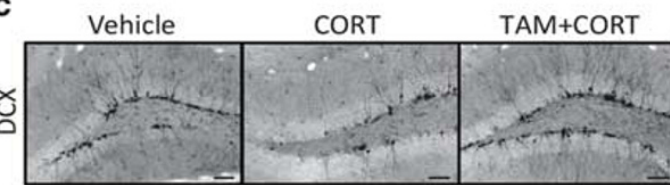

d
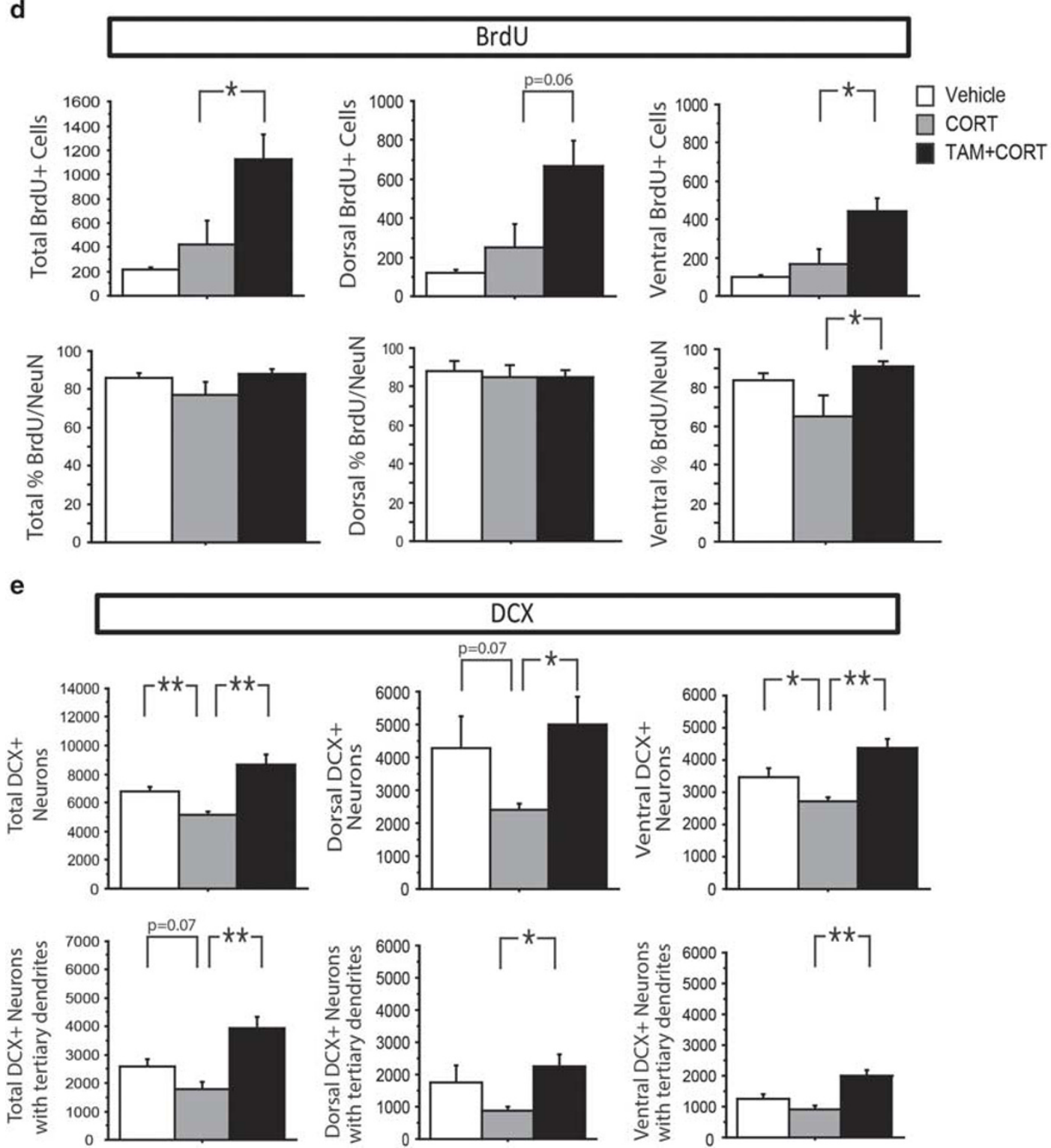
numbers of BrdU-labelled cells compared with CORT alone $(168.5 \%$ increase, $\mathrm{F}(1,9)=5.33, P<0.05)$ (Figure 3d), with similar effects in both dorsal and ventral dentate gyrus subregions.

Colabeling with NeuN was then used to determine the proportion of BrdU-labeled neurons in each group. While no differences were observed in the dorsal subregion, in the ventral dentate gyrus, TAM+CORT-treated animals were found to have a significantly higher percentage of cells colabeled for BrdU and NeuN than animals treated with CORT alone $(\mathrm{F}(1,8)=7.987, P<0.05)$ (Figure $3 \mathrm{~d})$.

To assess neurogenesis levels during behavioral testing, immunostaining was performed for the immature neuronal marker DCX, which labels cells that are a few days to about 3 weeks of age before sacrifice (Brown et al, 2003). We found that CORT treatment significantly decreased the number of DCX-positive cells compared with vehicle (24\% decrease, $\mathrm{F}(1,7)=15.994, P<0.01$ ) (Figure $3 \mathrm{e}$ ), while this was increased in the TAM+CORT group (69\% increase compared with CORT-alone group, $\mathrm{F}(1,10)=16.789, P<0.01)$. We next assessed the number of DCX-positive neurons with tertiary dendrites, which represent a more mature subset of adult-born granule cells. Here, there was a trend for an effect of CORT ( $31 \%$ decrease, $\mathrm{F}(1,7)=4.754, P=0.07)$, and a significant increase in TAM+CORT-treated mice $(120 \%$ increase compared with CORT-alone group, $\mathrm{F}(1,10)=$ 18.025, $P<0.01)$. When the data was split based on dorsal and ventral subregions, similar effects were seen in both subregions.

\section{Increased Adult Hippocampal Neurogenesis Promotes Resilience to the Behavioral Effects of Chronic CORT Administration, but Does Not Alter HPA Axis Activity}

After 4 weeks of CORT administration, mice were assessed on various behavioral tests of anxiety-related and antidepressant-responsive behavior. In some tests, there were no differences between any groups. For example, in the open field test, all groups behaved similarly in total distance, a measure of locomotive activity, as well as percent center distance, an anxiety measure (Fs $<1.755, P>0.05)$ (Figure $4 \mathrm{~b}$ ). However, the subset of behavioral tests that were affected by CORT, were also affected by an increase in neurogenesis (TAM+CORT group). In the elevated plus maze, CORT had an anxiogenic effect, manifested by decreased time spent in the open arms $(\mathrm{F}(1,25)=6.86, P<0.05)$ as well as fewer open arm entries $(\mathrm{F}(1,25)=6.547, P<0.05)$ (Figure $4 \mathrm{c}$ ), while this was reversed in TAM+CORT-treated mice $(\mathrm{F}(1,28)=6.273$, $P<0.05$ for open arm time, $\mathrm{F}(1,28)=4.252, P<0.05$ for open arm entries), showing that increasing adult hippocampal neurogenesis is sufficient to produce an anxiolytic effect in this test. In the tail suspension test, CORT decreased mobility as compared with vehicle $(\mathrm{F}(1,26)=5.13, P<0.05)$ (Figure $4 \mathrm{~d})$, and TAM+CORT treatment increased mobility $(\mathrm{F}(1,26)=$ 9.597, $P<0.01$ ), providing evidence that increased adult hippocampal neurogenesis is sufficient to promote stress resilience in this antidepressant-responsive test.

Along with the open field test, the forced swim and novelty suppressed feeding tests also showed no effect of CORT and a lack of an effect for TAM treatment (Fs $<2.419, P>0.05)$ (Supplementary Figure 1d and e). Consistent with the lack of an effect of increasing adult neurogenesis in the baseline, no- stress group, this suggests that neurogenesis may specifically affect behaviors that are impacted by chronic CORT in this paradigm.

Next, we were interested in assessing HPA axis regulation in these mice, to determine whether increasing neurogenesis prevents the effects of chronic CORT administration on HPA axis regulation. We have previously observed that our model of chronic CORT treatment has no effect on baseline CORT levels (unpublished data), however, does diminish the elevated levels of CORT produced in response to acute swim stress, as compared with control animals (David et al, 2009). Here, using the same paradigm, we observed a strong trend for decreased CORT levels following this acute swim stress in CORT-treated mice compared with controls $(\mathrm{F}(1,8)=5.155$, $P=0.05$ ) (Figure $4 \mathrm{e}$ ), and no difference between CORT and TAM+CORT-treated mice $(\mathrm{F}(1,9)=0.016, P>0.05)$, suggesting that both of these groups have similar impairment of the endogenous HPA axis response to acute stress. In the context of chronic CORT, we therefore observe a dissociation, where increased neurogenesis affects anxiety- and depressionrelated behavior, but not HPA axis regulation.

\section{DISCUSSION}

Here we have shown that increasing adult hippocampal neurogenesis is sufficient to promote resilience to chronic CORT administration. In iBax mice, we genetically block cell death in adult-born cells from neural precursors, which increases the number of adult-born neurons that reach maturation. In this way, we specifically mimicked this single property of antidepressants to increase adult neurogenesis, and showed that it is sufficient for antidepressant-like behavioral effects in tests that are sensitive to chronic CORT.

We show that chronic CORT has anxiogenic and depressive effects in a subset of behavioral tests, the elevated plus maze and the tail suspension test, which are prevented in mice with increased adult hippocampal neurogenesis (Figure 4). Conversely, in tests in which CORT has no effect, such as the open field test, increasing neurogenesis also has no effect. In this case, the behavior of all groups is similar to controls, and mimics the lack of an effect under baseline, nostress conditions (Figure 1). The disparity between the effects in these two situations, baseline and chronic CORT, suggests that in $i B a x$ mice, increased neurogenesis only affects anxiety and depression-related behavior when animals are in a stressed-like state.

Chronic CORT administration was used as a model of stress in these studies. While prior studies have observed effects of chronic CORT administration in different tests than observed here (David et al, 2009), we note that different strains of mice often display inconsistent behavioral phenotypes and responses to various stress models and drugs (Dulawa et al, 2004; Razzoli et al, 2011; Yalcin et al, 2008). In addition, as stress has many effects beyond CORT, which are not modelled here, additional studies would be necessary to fully understand how increased adult hippocampal neurogenesis interacts with stress.

A recent study found that BAX is required for long-term depression (LTD) but not basal synaptic transmission or long-term potentiation (Jiao and Li, 2011). Although the role of BAX in LTD was not examined in dentate granule 
a

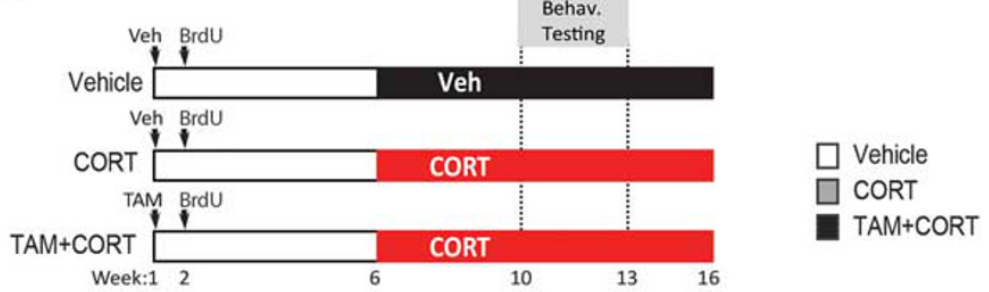

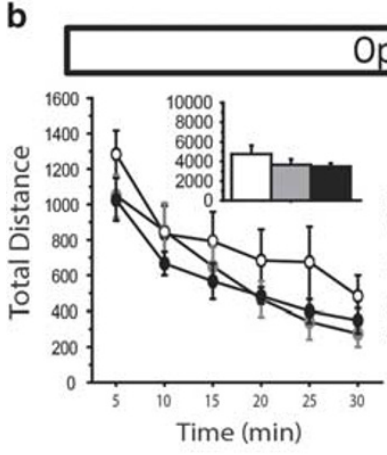

Open Field

d

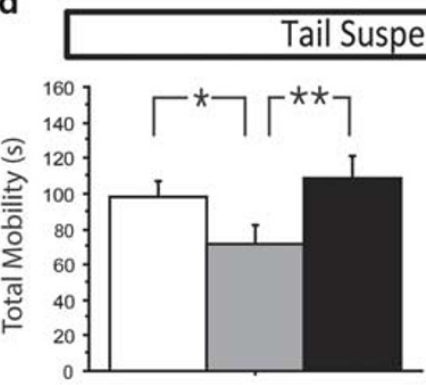

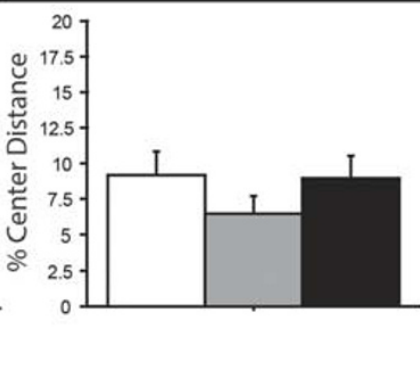

C

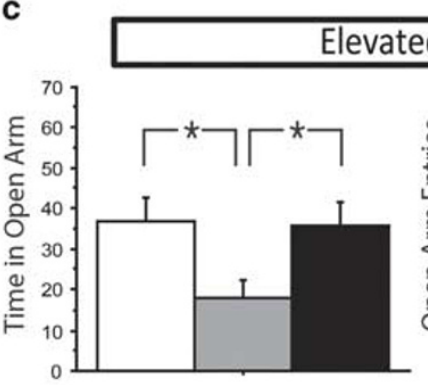

Elevated Plus Maze

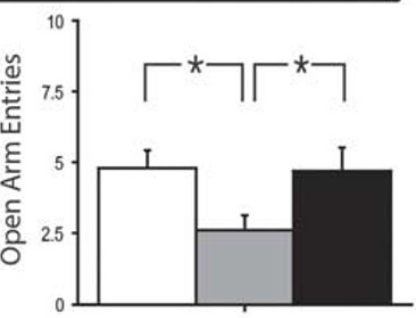

e

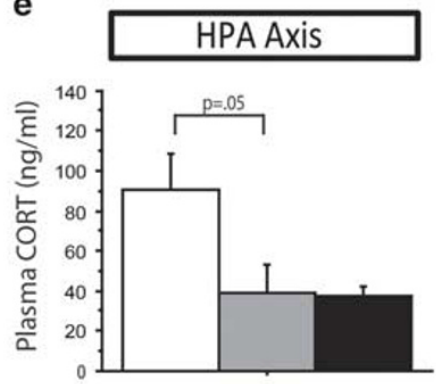

Figure 4 Genetically increasing adult hippocampal neurogenesis in iBax mice prevents the effects of chronic CORT on mood-related behavior, but does not affect HPA axis regulation. (a) Experimental timeline. (b) No statistically significant differences were seen between groups in total distance or percent center distance in the open field test $(P>0.05) . n=8-10 /$ group. (c) In the elevated plus maze, CORT-treated mice spent significantly less time in the open arms $(P=0.015)$ and had fewer open arm entries $(P=0.017)$ than controls. These effects were reversed in TAM+CORT-treated mice $(P=0.018$ for open arm time, $P=0.049$ for open arm entries). $n=12-15 /$ group. (d) In the tail suspension test, CORT-treated mice displayed decreased mobility $(P=0.032)$, which was reversed in TAM+CORT-treated mice $(P=0.005)$. In the line graph, data is represented in I-min bins for the duration of the test. $n=14-15 / g r o u p$. (e) Trunk blood was collected 5 min after a I-min swim stress, from which plasma was isolated. There is a strong trend for CORT-treated mice to have lower plasma CORT levels than controls following forced swim stress $(P=0.05)$, but no difference between CORT and TAM+CORT groups $(P=0.91)$. $n=5-6$ group. All error bars represent SEM. *P<0.05, **P<0.0I. CORT, corticosterone; HPA, hypothalamic-pituitary-adrenal; TAM, Tamoxifen.

neurons in that study, if this function is conserved across cell-types, including maturing adult-born neurons, then one cannot rule out the possibility that decreased LTD of adultborn neurons may contribute to some of the behavioral effects observed in this study. This is, however, unlikely, since the electrophysiological and behavioral impact of young neurons in $i B a x$ mice is consistent with increased neurogenesis rather than with altered properties (Sahay et al, 2011). Another potential concern is that the Nestin-CreERT2 driver line used here has been shown to drive ectopic expression of reporter lines (Sun et al, 2014), raising the possibility that LTD may be affected in additional cells of $i B a x$ mice. However, we have conducted additional studies utilizing the same Nestin-CreERT2 line to target adult-born neurons, where we see that $\mathrm{x}$-irradiation prevents the electrophysiological impact of these cells on hippocampal circuits, ruling out the possibility that ectopic expression through this driver line affects hippocampal activity (unpublished results). Nevertheless, we cannot rule out at present the possibility of ectopic effects in other brain regions.

One prevailing hypothesis in the literature is that adult neurogenesis affects anxiety- and depression-related behaviors through altered regulation of the HPA axis, since the absence of adult neurogenesis impairs HPA axis regulation following acute stress (Schloesser et al, 2009; Snyder et al, 2011) and prevents the effects of antidepressants on the HPA axis (Surget et al, 2011). While these studies suggest that intact adult hippocampal neurogenesis is required for proper HPA axis activity, our data suggest that increasing adult hippocampal neurogenesis is not sufficient to affect this process (Figures 2 and $4 \mathrm{e}$ ). While our findings do not 
fit into the framework laid out by the ablation studies cited above, our study is the first to test the HPA axis response using a model of increased, rather than decreased, adult hippocampal neurogenesis. Together, it appears that a certain level of neurogenesis is required for proper HPA axis regulation, but that levels above this threshold have no additive effect. While it is possible that increased neurogenesis may have more subtle effects on the HPA axis that were not observed here, our data suggest that increased neurogenesis likely can affect behavior through a mechanism independent of HPA axis regulation. Nevertheless, we do note that under different stress conditions, where the endogenous HPA axis is active, increased neurogenesis could affect behavior through an additional mechanism involving the HPA axis.

A previous study showed that the effects of social defeat stress and environmental enrichment on both neurogenesis and stress-related behavior are dependent on modulation of the HPA axis, as adrenalectomized mice with clamped CORT levels were unaffected by these environmental manipulations (Lehmann et al, 2013). Data presented here further suggests that CORT-mediated changes in neurogenesis are required for behavioral effects, as mice with increased neurogenesis do not display CORT-induced behavioral effects.

A recent study has shown that acute CORT treatment (for 7 days) shifts the balance of cell types produced towards fewer neurons and more oligodendrocytes, and has suggested the possibility that this shift may underlie behavioral effects of CORT treatment (Chetty et al, 2014). We note that here we did not observe a significant effect of CORT treatment on the percent of BrdU-positive cells that are colabeled with the neuronal marker NeuN (Figure 3d), possibly because BrdU was injected 4 weeks before the onset of CORT treatment (Figure 3a). However, we did observe a significant difference between CORT and CORT+TAM groups in the proportion of BrdU-positive cells colabeled with NeuN. This suggests that these two groups differ not only in the number of adult-born cells, but also in cell-types and functions.

It is particularly interesting that this difference is observed specifically in the ventral, but not dorsal, dentate gyrus. Numerous studies have now shown that the dorsal and ventral hippocampus have different gene expression, connectivity, and function (Fanselow and Dong, 2010; Kheirbek et al, 2013). In addition, many studies have observed larger effects of chronic stressors and antidepressants on ventral than dorsal neurogenesis (Tanti and Belzung, 2013b; Wu and Hen, 2014a; Wu et al, 2014b). Although we do not see large differences in neurogenesis levels between dorsal and ventral dentate gyrus, observation of this difference in proportion of adult-born neurons produced in the ventral dentate gyrus supports the hypothesis that neurogenesis in the ventral hippocampus may be particularly important for anxiety- and depression-related behavior.

While one identified function of the ventral hippocampus is to provide negative feedback to the HPA axis, primarily via connectivity through the bed nucleus of the stria terminalis (Cullinan et al, 1993; Herman et al, 1992), our data suggests that this process is not impaired by increasing adult hippocampal neurogenesis. We therefore suggest that the ventral hippocampus may mediate the behavioral effects observed here through a different mechanism, possibly through connectivity to other brain structures which receive ventral hippocampal input, such as the amygdala or nucleus accumbens (Canteras and Swanson, 1992; Fanselow and Dong, 2010; Namura et al, 1994)

While the studies presented here suggest that increasing adult hippocampal neurogenesis can protect against the behavioral effects of chronic exogenous CORT administration, it remains to be seen whether this manipulation can rescue behavior if neurogenesis is increased after chronic CORT. As antidepressants are generally prescribed after the onset of depression, this is an important point to be addressed for the translational feasibility of targeting neurogenesis. In addition, imaging tools being developed to detect levels of adult hippocampal neurogenesis in depressed patients may allow increased adult hippocampal neurogenesis to serve as a biomarker for antidepressant efficacy (Couillard-Despres and Aigner, 2011; Tanti and Belzung, 2013a).

Neural stem cells in the adult mammalian brain provide a resource that may be harnessed to treat various disorders. The experiments presented here suggest that expanding the production of adult-born neurons may be beneficial for the treatment of depression and anxiety disorders.

\section{FUNDING AND DISCLOSURE}

$\mathrm{RH}$ is supported by a US National Institute of Health grant R37MH068542, the National Institute of Aging grant R01AG043688, the Hope for Depression Research Foundation (HDRF), and the New York State Stem Cell Science (NYSTEM) contract CO26430. RH also receives compensation as a consultant for Lundbeck and Servier. AS is supported by a US National Institutes of Health grant 1R01MH104175, the Ellison Medical Foundation New Scholar in Aging, and a Whitehall Foundation grant. AS is also a consultant for Psybrain LLC. ASH declares no conflict of interest.

\section{ACKNOWLEDGMENTS}

We would like to thank K. Keener, M. Lauring, and C. Price for technical assistance.

\section{REFERENCES}

Batailler M, Droguerre M, Baroncini M, Fontaine C, Prevot V, Migaud M (2014). DCX-expressing cells in the vicinity of the hypothalamic neurogenic niche: a comparative study between mouse, sheep, and human tissues. J Comp Neurol 522: 1966-1985.

Boldrini M, Underwood MD, Hen R, Rosoklija GB, Dwork AJ, John Mann J et al (2009). Antidepressants increase neural progenitor cells in the human hippocampus. Neuropsychopharmacology 34: 2376-2389.

Brown JP, Couillard-Despres S, Cooper-Kuhn CM, Winkler J, Aigner L, Kuhn HG (2003). Transient expression of doublecortin during adult neurogenesis. J Comp Neurol 467: 1-10.

Canteras NS, Swanson LW (1992). Projections of the ventral subiculum to the amygdala, septum, and hypothalamus: a PHAL anterograde tract-tracing study in the rat. J Comp Neurol 324: 180-194. 
Chetty S, Friedman AR, Taravosh-Lahn K, Kirby ED, Mirescu C, Guo $\mathrm{F}$ et al (2014). Stress and glucocorticoids promote oligodendrogenesis in the adult hippocampus. Mol Psychiatry 19: $1275-1283$.

Couillard-Despres S, Aigner L (2011). In vivo imaging of adult neurogenesis. Eur J Neurosci 33: 1037-1044.

Cullinan WE, Herman JP, Watson SJ (1993). Ventral subicular interaction with the hypothalamic paraventricular nucleus: evidence for a relay in the bed nucleus of the stria terminalis. J Comp Neurol 332: 1-20.

David DJ, Samuels BA, Rainer Q, Wang JW, Marsteller D, Mendez I et al (2009). Neurogenesis-dependent and -independent effects of fluoxetine in an animal model of anxiety/depression. Neuron 62: 479-493.

Dayer AG, Ford AA, Cleaver KM, Yassaee M, Cameron HA (2003). Short-term and long-term survival of new neurons in the rat dentate gyrus. J Comp Neurol 460: 563-572.

Denny CA, Burghardt NS, Schachter DM, Hen R, Drew MR (2012). 4- to 6-week-old adult-born hippocampal neurons influence novelty-evoked exploration and contextual fear conditioning. Hippocampus 22: 1188-1201.

Dranovsky A, Picchini AM, Moadel T, Sisti AC, Yamada A, Kimura $S$ et al (2011). Experience dictates stem cell fate in the adult hippocampus. Neuron 70: 908-923.

Dulawa SC, Holick KA, Gundersen B, Hen R (2004). Effects of chronic fluoxetine in animal models of anxiety and depression. Neuropsychopharmacology 29: 1321-1330.

Duman RS, Nakagawa S, Malberg J (2001). Regulation of adult neurogenesis by antidepressant treatment. Neuropsychopharmacology 25: 836-844.

Fanselow MS, Dong HW (2010). Are the dorsal and ventral hippocampus functionally distinct structures? Neuron 65: 7-19.

Gould E, McEwen BS, Tanapat P, Galea LA, Fuchs E (1997). Neurogenesis in the dentate gyrus of the adult tree shrew is regulated by psychosocial stress and NMDA receptor activation. J Neurosci 17: 2492-2498.

Herman JP, Cullinan WE, Young EA, Akil H, Watson SJ (1992). Selective forebrain fiber tract lesions implicate ventral hippocampal structures in tonic regulation of paraventricular nucleus corticotropin-releasing hormone $(\mathrm{CRH})$ and arginine vasopressin (AVP) mRNA expression. Brain Res 592: 228-238.

Jiao S, Li Z (2011). Nonapoptotic function of BAD and BAX in long-term depression of synaptic transmission. Neuron 70: 758-772.

Kee N, Teixeira CM, Wang AH, Frankland PW (2007). Preferential incorporation of adult-generated granule cells into spatial memory networks in the dentate gyrus. Nature neuroscience 10: $355-362$.

Kheirbek MA, Drew LJ, Burghardt NS, Costantini DO, Tannenholz L, Ahmari SE et al (2013). Differential control of learning and anxiety along the dorsoventral axis of the dentate gyrus. Neuron 77: 955-968.

Kokoeva MV, Yin H, Flier JS (2005). Neurogenesis in the hypothalamus of adult mice: potential role in energy balance. Science 310: 679-683.

Lee DA, Blackshaw S (2012). Functional implications of hypothalamic neurogenesis in the adult mammalian brain. Int $J$ Dev Neurosci 30: 615-621.

Lehmann ML, Brachman RA, Martinowich K, Schloesser RJ, Herkenham M (2013). Glucocorticoids orchestrate divergent effects on mood through adult neurogenesis. J Neurosci 33: 2961-2972.

Li Y, Li Y, McKay RM, Riethmacher D, Parada LF (2012). Neurofibromin modulates adult hippocampal neurogenesis and behavioral effects of antidepressants. J Neurosci 32: 3529-3539.

Malberg JE, Eisch AJ, Nestler EJ, Duman RS (2000). Chronic antidepressant treatment increases neurogenesis in adult rat hippocampus. J Neurosci 20: 9104-9110.
Namura S, Takada M, Kikuchi H, Mizuno N (1994). Topographical organization of subicular neurons projecting to subcortical regions. Brain Res Bull 35: 221-231.

Pierce AA, Xu AW (2010). De novo neurogenesis in adult hypothalamus as a compensatory mechanism to regulate energy balance. J Neurosci 30: 723-730.

Razzoli M, Carboni L, Andreoli M, Ballottari A, Arban R (2011). Different susceptibility to social defeat stress of BalbC and C57BL6/J mice. Behav Brain Res 216: 100-108.

Saaltink DJ, Havik B, Verissimo CS, Lucassen PJ, Vreugdenhil E (2012). Doublecortin and doublecortin-like are expressed in overlapping and non-overlapping neuronal cell population: implications for neurogenesis. J Comp Neurol 520: 2805-2823.

Sahay A, Scobie KN, Hill AS, O'Carroll CM, Kheirbek MA, Burghardt NS et al (2011). Increasing adult hippocampal neurogenesis is sufficient to improve pattern separation. Nature 472: 466-470.

Santarelli L, Saxe M, Gross C, Surget A, Battaglia F, Dulawa S et al (2003). Requirement of hippocampal neurogenesis for the behavioral effects of antidepressants. Science 301: 805-809.

Schloesser RJ, Lehmann M, Martinowich K, Manji HK, Herkenham M (2010). Environmental enrichment requires adult neurogenesis to facilitate the recovery from psychosocial stress. Mol Psychiatry 15: 1152-1163.

Schloesser RJ, Manji HK, Martinowich K (2009). Suppression of adult neurogenesis leads to an increased hypothalamo-pituitaryadrenal axis response. Neuroreport 20: 553-557.

Snyder JS, Soumier A, Brewer M, Pickel J, Cameron HA (2011). Adult hippocampal neurogenesis buffers stress responses and depressive behaviour. Nature 476: 458-461.

Sun MY, Yetman MJ, Lee TC, Chen Y, Jankowsky JL (2014). Specificity and efficiency of reporter expression in adult neural progenitors vary substantially among nestin-CreER(T2) lines. J Comp Neurol 522: 1191-1208.

Surget A, Tanti A, Leonardo ED, Laugeray A, Rainer Q, Touma C et al (2011). Antidepressants recruit new neurons to improve stress response regulation. Mol Psychiatry 16: 1177-1188.

Surget A, Wang Y, Leman S, Ibarguen-Vargas Y, Edgar N, Griebel G et al (2009). Corticolimbic transcriptome changes are state-dependent and region-specific in a rodent model of depression and of antidepressant reversal. Neuropsychopharmacology 34: 1363-1380.

Takeuchi O, Fisher J, Suh H, Harada H, Malynn BA, Korsmeyer SJ (2005). Essential role of BAX,BAK in B cell homeostasis and prevention of autoimmune disease. Proc Natl Acad Sci USA 102: 11272-11277.

Tanti A, Belzung C (2013a). Hippocampal neurogenesis: a biomarker for depression or antidepressant effects? Methodological considerations and perspectives for future research. Cell Tissue Res 354: 203-219.

Tanti A, Belzung C (2013b). Neurogenesis along the septo-temporal axis of the hippocampus: are depression and the action of antidepressants region-specific? Neuroscience 252: 234-252.

Toni N, Teng EM, Bushong EA, Aimone JB, Zhao C, Consiglio A et al (2007). Synapse formation on neurons born in the adult hippocampus. Nat Neurosci 10: 727-734.

van Praag H, Shubert T, Zhao C, Gage FH (2005). Exercise enhances learning and hippocampal neurogenesis in aged mice. J Neurosci 25: 8680-8685.

Walker AK, Rivera PD, Wang Q, Chuang JC, Tran S, OsborneLawrence $S$ et al (2014). The P7C3 class of neuroprotective compounds exerts antidepressant efficacy in mice by increasing hippocampal neurogenesis. Mol Psychiatry 20: 500-508.

Wang G, Han T, Nijhawan D, Theodoropoulos P, Naidoo J, Yadavalli S et al (2014). P7C3 neuroprotective chemicals function by activating the rate-limiting enzyme in NAD salvage. Cell 158: 1324-1334.

Wu MV, Hen R (2014a). Functional dissociation of adult-born neurons along the dorsoventral axis of the dentate gyrus. Hippocampus 24: 751-761. 
Wu MV, Shamy JL, Bedi G, Choi CW, Wall MM, Arango V et al (2014b). Impact of social status and antidepressant treatment on neurogenesis in the baboon hippocampus. Neuropsychopharmacology 39: 1861-1871.

Xu Y, Tamamaki N, Noda T, Kimura K, Itokazu Y, Matsumoto N et al (2005). Neurogenesis in the ependymal layer of the adult rat 3rd ventricle. Exp Neurol 192: 251-264.
Yalcin I, Belzung C, Surget A (2008). Mouse strain differences in the unpredictable chronic mild stress: a four-antidepressant survey. Behav Brain Res 193: 140-143.

Yin TC, Britt JK, De Jesus-Cortes H, Lu Y, Genova RM, Khan MZ et al (2014). P7C3 neuroprotective chemicals block axonal degeneration and preserve function after traumatic brain injury. Cell Rep 8: 1731-1740.

Supplementary Information accompanies the paper on the Neuropsychopharmacology website (http://www.nature.com/npp) 